\title{
PENSATA
}

Submetida 01.12.2019. Aprovada 22.04.2020

Avaliado pelo sistema double blind review. Editor Científico: Eduardo Ayrosa

Versão original

DOI: http://dx.doi.org/10.1590/So034-759020200406

\section{REDES DE DESEJO OU DE GOZO? EXPERIÊNCIA DE CONSUMO E NOVOS AGENCIAMENTOS TECNOLÓGICOS}

No debate internacional dos estudos do consumidor, a literatura sobre "experiência de consumo" já conta mais de três décadas, desde que foi sintetizada a partir dos estudos seminais de Hirschman e Holbrook (1982), sobre consumo hedônico; e de Holbrook e Hirschman (1982), sobre os aspectos experienciais do consumo. Tais estudos visavam definir a experiência de consumo como busca de diversão, fantasia, excitação sensorial e prazer, resultando em uma experiência mágica, extraordinária, memorável.

A importância dos aspectos estéticos, simbólicos e emocionais, nos estudos do consumidor, remonta à década de 1950, com os trabalhos de Gardner e Levy (1955), Levy (1959), Levy e Czepiel (1974), entre outros. Mas a temática da experiência de consumo só se tornou dominante quando os textos de Hirschman e Holbrook argumentaram a favor do resgate dos sentimentos e emoções envolvidos no processo de consumo. Holbrook (2006, p. 259) até mesmo criticou a quantidade de publicações que não mencionavam as pesquisas sobre o tema já produzidas, insistindo que a temática da experiência de consumo já estava presente em análises como as de Alderson (1957) e Abott (1955), e sugerindo que as raízes do conceito poderiam remontar às contribuições de clássicos da Economia, conforme mencionado no tratado sobre a felicidade de Lebergott (1993). Mesmo assim, os textos de Holbrook e Hirschman foram repetidamente citados como fundadores de um novo momento no qual a experiência de consumo havia se tornado "um elemento-chave na compreensão do comportamento do consumidor e, para alguns, uma base para a economia e o marketing do futuro" (Carù \& Cova, 2003, p. 267).

Embora as raízes do conceito de "experiência de consumo" sejam anteriores aos anos 1980, algo estava acontecendo, nesse contexto histórico, que ressignificou o debate em torno dessa temática e transformou os artigos de Hirschman e Holbrook em uma espécie de textos fundadores para o campo dos estudos do consumidor e do marketing. Refiro-me ao momento em que um novo tipo de vida social começava a ganhar forma, de um lado, resultado de uma nova ordem econômica e, de outro, de um novo tipo de experiência de tempo-espaço, nomeada pelo crítico cultural americano Fredric Jameson "experiência pós-moderna". A emergência de uma "sociedade pós-industrial ou do consumo; da mídia ou do espetáculo; ou do capitalismo multinacional” (Jameson, 1998, p. 3) era correlata de uma virada cultural que transformou o consumo em "um ato de produção de experiências "(Firat \& Dholakia, 1998, p. 96). Nesse cenário, consumir experiências torna-se uma nova abordagem no campo dos estudos do consumidor e do marketing (Carù \& Cova, 2003; Gilovich, Kumar, \& Jampol, 2015; Jantzen, Fitchett, Ostegaard, \& Vetner, 2012; Lanier \& Rader, 2015; Pine \& Gilmore, 1999; Schmitt, 1999; Woodward \& Holbrook, 2013).

1 Fundação Getulio Vargas, Escola de Administração de Empresas de São Paulo, São paulo, SP, Brasil

FONTENELLE isleide.fontenelle@fgv.br ORCID: 0000-0001-5607-324X 
Os artigos de Hirschman e Holbrook não destacam a importância do contexto histórico da década de 1980 na proposição que fazem da noção de experiência de consumo, mas os autores deixam entrever a importância desse novo momento quando demonstram interesse em discutir os aspectos experienciais do consumo a partir de uma classe específica de produtos mais aderente, segundo eles, ao conceito que propõem, dada a capacidade desses produtos de gerar um forte envolvimento emocional, a saber, os produtos culturais e estéticos. Os autores estavam interessados em apresentar os limites do "information processing model" - considerado o modelo hegemônico da pesquisa do consumidor -, para a compreensão do novo tipo de consumo que emergia. Assim, nos dois referidos artigos, acabaram por condensar dois objetivos diretamente relacionados ao debate da experiência de consumo, propondo, por um lado, uma compreensão dessa categoria de forma epistemológica e metodologicamente diferente do modelo dominante e, por outro, mobilizando o conceito para a compreensão de uma classe específica de produtos. E, mesmo que tenham insistido que "todos os produtos podem ser hedonicamente experienciados pelos consumidores" (Hirschman \& Holbrook, 1982, p. 96), a ênfase que deram ao consumo de bens imateriais acabou por gerar certa sobreposição conceitual entre dois campos de análise que se abrem a partir da perspectiva da experiência de consumo: de um lado, a compreensão da "experiência de consumo" como constitutiva de qualquer prática de consumo e, de outro, o consumo de experiência como um tipo específico de consumo no qual se vende uma concepção de experiência como mercadoria (Campbel, 2007; Lanier \& Rader, 2015).

Em busca de uma diferenciação que consideravam necessária para a "experiência de consumo", Carù e Cova (2003) atribuíram-lhe uma concepção mais sociológica, na qual caberiam as experiências ordinárias, simples, vividas fora do mercado, quais sejam aquelas que desfrutamos na vida em família, com amigos ou como cidadãos. Como contraponto, propuseram o conceito de "consumer experience", significando as experiências produzidas pelo mercado, particularmente, aquelas que visam o extraordinário e o memorável, foco do "marketing experiencial”. Mas para Holbrook, em uma entrevista que deu a Woodward, essa diferenciação não fazia sentido, já que a "experiência de consumo" definiria toda a nossa experiência de vida (Woodward \& Holbrook, 2013).

A meu ver, a "experiência de consumo" já nasce como uma categoria mercadológica, e não faz sentido extrapolar o seu significado para além dos muros do mercado. Mesmo que Holbrook tenha enfatizado que há experiências fora do mercado, o termo "experiência de consumo" já traz sua marca de nascença, no sentido de que ressignificou a concepção de experiência para o contexto dos estudos do consumidor e do marketing. E se a proposição dessa categoria, conforme Holbrook, gerou resistência no campo acadêmico por parte daqueles que ainda insistiam nos "bons velhos tempos, quando estávamos preocupados com o comportamento do comprador" (Woodward \& Holbrook, 2013, p. 326), por outro lado, o termo também chegou como renovação para um campo no qual a compreensão do consumidor como um ser apenas racional já não era suficiente. Além disso, a categoria "experiência de consumo" acabou dando forma a um novo modo de atuação do mercado, baseado na "venda de experiências". Mesmo insistindo que não foi essa a sua intenção, Holbrook admite que a ideia de experiências de consumo alimentou uma tendência em direção ao marketing experiencial.

Não por acaso, quando Pine e Gilmore (1999) propõem um novo tipo de economia da experiência, eles argumentam que as sensações e emoções envolvidas nas experiências de consumo já existiam, mas elas ainda não haviam sido compreendidas como capazes de gerar valor. Ao escreverem um livro que, segundo Holbrook (2006, p. 259), seguiu o estilo das melhores práticas da Harvard Business Review, os autores visaram novas formas de agregar valor às empresas, focando as diversas oportunidades de negócios que se abrem quando se comercializam sensações e emoções. Assim, Pine e Gilmore conseguem ir além das análises acadêmicas iniciais de Holbrook e Hirshman, pois, enquanto esses estavam ocupados em resgatar o foco perdido em marketing acerca da experiência no consumo, aqueles já estavam voltados à transformação da experiência em mercadoria, estágio para o qual o conceito de "experiência de consumo" serviu bem. Voltando à afirmação de Holbrook de que "TODAS as experiências são experiências de consumo e que todas essas experiências de consumo constituem a maior parte do que fazemos durante nossas vidas de vigília e até não-vigília” (Woodward \& Holbrook, 2013, p. 325 - destaque do autor), sua frase foi premonitória de um tempo, o nosso, no qual é o mercado que tem investido na transformação de toda experiência humana em experiência de consumo.

“Experiência de consumo" contém uma palavra complexa, experiência, cujo significado não pode ser compreendido sem que se elabore uma importante digressão sobre a que tradição do pensamento o conceito pertence (Carù \& Cova, 2003; Jay, 1994; Lasch, 2006). Mas Holbrook e Hirschman (1982) não propõem uma definição de experiência. Eles criam uma nova categoria-conceito, a "experiência de consumo", dando a ela um significado que, segundo os autores, estava relacionado a uma "perspectiva experiencial” que é "fenomenológica em 
espírito e considera o consumo como estado de consciência principalmente subjetivo, com uma variedade de significados simbólicos, respostas hedônicas e critérios estéticos” (p. 132). $\mathrm{Na}$ indicação das bases teóricas dessa categoria, os autores mencionam, embora não aprofundem, o conceito freudiano do “princípio do prazer”, enfatizando sua importância para uma “perspectiva experiencial”, voltada a entender o consumo como busca de satisfação, entendida como diversão e prazer. Mas, se seguirmos a análise freudiana, o princípio do prazer não esgota a compreensão da busca da experiência de satisfação. Por isso, o psicanalista desenvolveu sua concepção de um “além do princípio do prazer" (Freud, 1996), no sentido de se referir a uma busca incessante de satisfação, compreendida como uma compulsão à repetição que nem sempre seria sinônimo de prazer ou que poderia encontrar prazer no desprazer. Certamente, há uma série de gradações entre esses dois polos. Voltaremos a esse ponto porque ele é central no argumento que proponho desenvolver neste ensaio.

Antes, porém, é importante destacar que foi a partir da ideia de experiências prazerosas, memoráveis e extraordinárias que se delimitou todo um campo de pesquisa em torno do conceito de experiência de consumo, embora algumas vozes críticas apontassem para a necessidade de se considerarem os riscos dos excessos a que a ideia de extraordinário poderia levar (Carù \& Cova, 2003). E análises recentes apontam para tipos de consumo excessivos, vividos sob a forma de dor (Scott, Cayla, \& Cova, 2017) ou carregados de negatividade (Raymen \& Smith, 2017; Smith \& Raymen, 2017), desafiando a concepção positiva da "experiência de consumo"e trazendo novas questões para a ideia de experiências prazerosas e extraordinárias (Arnould \& Price, 1993; Belk \& Costa, 1998; Kozinets, 2002) ou até mesmo para o entendimento do que são as experiências ordinárias de consumo (Carù \& Cova, 2003).

Nesse cenário, que corresponde a uma expansão mercadológica que promete experiências sem fim (Loose, 2015) e às transformações tecnológicas que indicam um aumento da nossa paixão pelo consumo que também puxa para extremos (Kozinetes, Patterson, \& Ashman, 2017), considero que o conceito de experiência de consumo é necessário para se pensar esse apelo excessivo a um tipo de satisfação que pode também se tornar excessiva. No entanto, as pesquisas sobre formas de consumo excessivo não fazem uso do conceito de experiência de consumo, que ficou circunscrito apenas às análises do universo mágico do consumo e de suas formas prazerosas. São duas correntes temáticas que não dialogam, como se pertencessem a campos epistemológicos e teóricos distintos. O objetivo deste meu ensaio é propor uma relação entre essas duas formas de consumo, no sentido de argumentar que o consumo excessivo, analisado nesses estudos, aponta para a mesma busca de satisfação que está na concepção da experiência de consumo proposta por Holbrook e Hirshman; e que a diferença pode ser apenas de grau. Para isso, proponho entendermos experiência de consumo como busca de satisfação pulsional ou de gozo. Este ensaio inicia-se, portanto, discorrendo sobre o que é a satisfação pulsional, conceito freudiano que foi posteriormente alargado por Lacan (1998) a partir da categoria de gozo (jouissance). Tal percurso teórico é necessário para que possamos entender o papel que certas formas culturais têm na contenção ou na convocação a essa busca de satisfação/ gozo. Veremos como, ao longo do século XX, a cultura do consumo foi se transformando em um locus privilegiado de convocação ao gozo, em especial, a partir das décadas finais do século XX, com o surgimento de novas tecnologias e a ascensão de uma era digital. É nesse cenário que a convocação excessiva a uma experiência de consumo, também excessiva, começa a florescer. Para ilustrar a teoria, tomarei alguns exemplos empíricos, particularmente, a pesquisa realizada por Kozinets et al. (2017) sobre a paixão pelo consumo no compartilhamento de comida on-line. E, na medida em que tal pesquisa se apoia na teoria deleuziana do desejo e na sua concepção de agenciamento - entendido como a própria rede, a articulação entre corpos, máquinas, algoritmos, enunciados, afetos, entre outros -, o ensaio se encerra propondo um necessário “encontro" entre a noção psicanalítica de pulsão e a de desejo em Deleuze (David-Ménard, 2014; Zizek, 2011). Os agenciamentos tecnológicos podem ser compreendidos como uma forma de convocação ao gozo que a cultura do consumo contemporânea vem promovendo. Dessa forma, mais do que um aprofundamento ou contribuição a um campo teórico específico, este ensaio visa contribuir propondo certas possibilidades de análise ao campo dos estudos críticos do consumo. De um lado, propõe a articulação entre a experiência de consumo e o consumo excessivo, por meio dos conceitos de pulsão e de gozo; de outro, argumenta que o conceito de satisfação pulsional/gozo tem muito a contribuir para uma perspectiva crítica aos estudos contemporâneos sobre consumo e novos agenciamentos tecnológicos que vêm sendo apoiados na teoria deleuziana do desejo.

\section{Experiência de consumo como busca de satisfação pulsional ou busca de gozo}

Retorno a quando mencionei o uso da teoria freudiana do princípio do prazer nos estudos iniciais de Holbrook e Hirschman sobre a experiência de consumo. Como já dito, não há como discorrer sobre o princípio do prazer freudiano sem abordar sua proposição de um 
“além do princípio do prazer", pois, para o psicanalista, a busca humana por satisfação resultaria, sempre, em uma satisfação paradoxal: parcial e momentânea, podendo não resultar em prazer e apelando sempre a um excesso, tendo na cultura o seu anteparo. Essa busca por satisfação foi nomeada por Freud pulsional.

Pulsão é um dos conceitos mais importantes e complexos da metapsicologia freudiana, referindo-se a algo que opera numa zona de indeterminação, de indistinção entre corpo e aparelho psíquico, pois sua fonte é sempre somática, mas ela só se torna acessível por meio do seu representante psíquico (Freud, 2014a, 2014b; Ianninni, 2014). Segundo Zizek (2011), foi a teoria freudiana que primeiro levantou a questão fundamental do corpo “erotizado, sustentado pela libido, organizado ao redor de zonas erógenas, não animalístico e não biológico” (p. 137). Daí porque esse corpo pulsional se torna o objeto da psicanálise, um corpo “enquanto superfície para a inscrição dos traços de traumas e prazeres excessivos, o corpo através do qual o inconsciente fala" (p. 138). Nos artigos finais que compõem o compêndio de psicanálise (Freud, 2014b), o conceito de pulsão ganha um estatuto epistemológico central, de onde derivariam todos os demais, por exemplo, o de inconsciente. Freud considerou a pulsão como o objeto principal da psicanálise porque ela é determinante para o modo humano de desejar, de ter prazer e de sofrer, envolvendo um corpo formado e transformado pela cultura.

A pulsão é caracterizada como uma força constante oriunda do interior do corpo, sem oferecer nenhuma possibilidade de fuga do sujeito desse estado de tensão. A remoção da excitação interna, o alívio da tensão, é o que visa a pulsão para satisfazer-se. Mas, como essa força é constante, essa satisfação é sempre provisória, e o alívio sentido como satisfação pulsional pode também acarretar sensações consideradas desprazerosas. Foi diante desse paradoxo que Freud elaborou o seu conceito de “além do princípio do prazer" (Freud, 1996), a fim de apontar para algo que leva o sujeito além de um prazer não vinculado a formas culturalmente determinadas como objetos de satisfação. Estar além do prazer significa que o sujeito pode ser levado a “não buscar seu próprio bem” (Copjec, 1994, p. 87). É a partir do desenvolvimento do conceito de "além do princípio do prazer" que as denominações iniciais de Freud acerca das pulsões pulsões do eu ou de autopreservação e pulsões sexuais (Freud, 2014a) - se modificam, sendo renomeadas como pulsões de vida e de morte. Para os propósitos deste ensaio, interessa-nos aprofundar a noção de pulsão de morte, seja pela associação que Freud fez entre esse conceito e a "compulsão à repetição", seja pela maneira como o conceito foi absorvido e aprofundado por Lacan (1998) e que permite, por sua vez, sua conexão com a cultura contemporânea do consumo.
Jacques Lacan retomou a problemática freudiana do "além do princípio do prazer" a partir da categoria do gozo. Assumindo que "o uso da função da pulsão não tem outro valor senão o de pôr em questão o que é da satisfação” (Lacan, 1998, p. 158), o psicanalista francês define pulsão como busca de gozo, uma busca que sempre deixa um saldo de insatisfação. 0 autor insiste na impossibilidade de um objeto empírico capaz de satisfazer plenamente a pulsão que, "apreendendo seu objeto, aprende de algum modo que não é justamente por aí que ela se satisfaz" (Lacan, 1998, p. 159).

Portanto, a pulsão está sempre contornando um objeto inalcançável, configurado como um resto de gozo a recuperar. 0 gozo pode ser compreendido como a satisfação impossível que toda pulsão visa, por isso, para Lacan, só haveria uma pulsão, que é a pulsão de morte, já que mesmo a libido - que foi considerada por Freud como pulsão de vida - estaria nesse circuito repetitivo, nessa compulsão à repetição, em busca de uma satisfação impossível. Em sintonia com essa perspectiva, Zizek (2011) argumenta que a pulsão de morte freudiana, no sentido do "além do princípio do prazer”, significa a radical “insistência de um organismo em repetir infinitamente o estado de tensão" (p. 46).

É a instância simbólica que barra o acesso ao gozo e, assim, permite a emergência do sujeito do desejo, mantendo a coerência imaginária do eu. Ou seja, para desejar, é preciso aceitar uma perda de gozo. Se a pulsão não pode satisfazer-se plenamente, é porque toda a montagem das pulsões e seus destinos estão em permanente relação com a inscrição pulsional no registro da cultura, compreendida como lugar da lei e da linguagem. Por isso mesmo, Freud desenvolveu a sua tese do mal-estar na civilização a partir de um aforisma central: o de que a civilização resulta da renúncia à satisfação direta das pulsões (Freud, 2011). Lacan insiste nessa perspectiva, indicando a instância simbólica como barreira ao gozo e operação necessária para a emergência do sujeito do desejo. 0 gozo só é acessível à medida que o sujeito se perde como tal. Não é por acaso que "a emergência do que é a da ordem da pulsão aparece constantemente em Lacan envolto na temática de um gozo que flerta com o informe; gozo para além do princípio do prazer que é, no fundo, gozo para além do princípio de submissão a representações" (Safatle, 2007, p. 170). Daí Lacan afirmar que "o caminho da pulsão é a única forma de transgressão que se permite ao sujeito em relação ao princípio do prazer" (Lacan, 1998, p. 174). A satisfação que a pulsão alcança, sempre parcial, é a única via possível para uma forma de satisfação que não abole o ser. Por isso, a pulsão pode tomar muitos destinos e variar de objetos, em busca de algo que é invariável: sua satisfação. A barreira ao gozo constitui, assim, o sujeito do desejo que, apoiado no princípio do prazer, fantasia 
uma satisfação plena, buscando, repetidamente, objetos e satisfações substitutivas para aquela satisfação/gozo impossível, entre os quais os objetos da cultura do consumo.

A teoria pulsional torna-se, desse modo, pertinente aos estudos relacionados à temática da "experiência de consumo", na medida em que a busca de satisfação pulsional é constitutiva de qualquer forma de consumo - comercial ou não comercial, material ou imaterial, sólida ou líquida (Bardhi \& Eckhardt, 2017). Por isso mesmo, considero a busca de satisfação pulsional a matéria-prima da cultura do consumo desde que as pesquisas do consumidor e o marketing se firmaram como campos de saber e de ação voltados a compreender "o que quer o consumidor". Afinal, os estudos do consumidor sempre visaram entender, para fornecer, o que o consumidor busca, a saber, uma satisfação sensorial, estética, psíquica. Já vimos que foi com esse objetivo que o conceito da "experiência de consumo" emergiu, aportando apenas experiências consideradas “prazerosas" no reino extraordinário e positivo do consumo. 0 que aconteceria, porém, a uma cultura, e aos seus sujeitos, se, ao invés de barrar, essa cultura estimulasse experiências de consumo excessivas?

\section{Gestão de gozo pelo consumo: a forma cultural como operadora da satisfação pulsional}

A teoria das pulsões raramente foi utilizada de maneira direta no campo dos estudos do consumo. Encontramos a exceção em Loose (2015), que faz uma análise do uso da arte pelo anúncio comercial com base nas relações entre pulsão, consumo e excesso. 0 autor problematiza o quanto a relação entre ciência e mercado tem buscado garantir satisfações além do princípio do prazer. Trata-se, segundo ele, de uma lógica que opera por meio da adicção, que se tornou uma resposta contemporânea à busca da satisfação pulsional por meio do consumo.

Na teoria freudiana da pulsão, a cultura sempre foi o anteparo ao excesso. Mas é importante não confundir cultura como instância simbólica - na qual a proibição ao gozo é estrutural - com formas culturais que se desenvolvem sob essa instância (Lacan, 1998). Nossa forma cultural estimula a produção e o consumo excessivos, assim como promove a insatisfação de modo a que se consuma constantemente. Nós estamos em uma sociedade de "gozo comandado", na excelente expressão de McGowan (2004, p. 7), cuja lógica de funcionamento organiza-se a partir de convocações ao gozo, sob a forma-mercadoria. Essa forma cultural é resultado direto de um estímulo ao consumo que se intensificou na segunda metade do século XX e que ganhou novo impulso com as inovações tecnológicas.
Esse empuxo ao excesso por meio de múltiplas convocações ao gozo já vem sendo objeto de análise de estudiosos do campo do consumo, seja na forma de consumo material, como em casos de violência em experiências extremas de compras (Smith \& Raymen, 2017); seja em formas específicas de consumo experiencial, por meio da estranha busca por sentir dor (Scott et al., 2017); seja no vício oriundo das novas modalidades de consumo tecnológico, tais como na relação entre jogos eletrônicos e lazer desviante (Raymen \& Smith, 2017), ou no fenômeno de compartilhamento de imagens de comida em redes sociais (Kozinets et al., 2017). Embora tais abordagens não se utilizem da teoria freudiana das pulsões, algumas delas se sustentam em campos teóricos que derivam diretamente de tal perspectiva (Raymen \& Smith, 2017; Smith \& Raymen, 2017) ou estão em relação com ela (Kozinets et al., 2017). No primeiro caso, os autores, apoiados na abordagem lacaniana do gozo, a partir da interpretação de Slavoj Zizek (2002), analisaram as relações entre capitalismo, consumo e excesso. Já Kozinets et al. (2017) basearam-se na teoria do desejo de Deleuze e Guattari (1983, 1987) para discutirem como a tecnologia tem aumentado o nosso desejo pelo consumo, puxando-o para extremos.

Esses estudos ilustram bem o modo de convocações ao gozo da cultura do consumo contemporânea. Kozinets et al. (2017) revelam essas convocações sob a forma de novos agenciamentos tecnológicos a partir dos quais a experiência de consumo material tem sido, cada vez mais, impulsionada pelo virtual, ao mesmo tempo que também o alimenta. Há uma imbricação entre essas formas de consumo por meio dos fluxos de dados e paixões que circulam entre o mundo virtual e o real, comandada pelos interesses de um capitalismo tecnológico que, por sua vez, conta com "altos níveis de envolvimento apaixonado do consumidor" (Kozinets et al., 2017, p. 678). Os autores mapearam aquilo que nomeiam circuito de produção de desejos - de consumo de comida ou de suas imagens -, no qual consumidores, softwares, algoritmos e corporações estão envolvidos em um complexo e dinâmico sistema de feedback que resulta em um movimento constante de circulação de paixões consumistas. Exemplificam: os consumidores compartilham seus desejos por um tipo particular de comida por meio de uma imagem. Outros consumidores reagem a isso. Softwares e algoritmos entram em ação e promovem outras conexões envolvendo corporações ligadas à comida, desde o agrobusiness até supermercados, lojas especializadas e menus de restaurantes. Novas imagens e produtos são oferecidos, despertando novos desejos de consumo, em um circuito infinito. Trata-se de um processo no qual as redes sociais têm tido um papel fundamental na organização e convocação das nossas paixões/pulsões. 
No caso específico da busca por satisfação por meio da produção e consumo de imagens de comida, os autores identificaram um empuxo para o excesso, nominado "food porn”, compreendendo pornográfico como o que é excessivo. Tal excesso pode ocorrer tanto em seu sentido físico quanto imagético. Mas há um limite biológico real para o quanto podemos comer que, se ultrapassado, faz o corpo padecer. Já o virtual promove um consumo sem limites, prometendo libertar o corpo dos seus constrangimentos físicos, veiculando imagens de comida - ordinárias ou extremas - e canalizando um fluxo de desejos que acaba sendo mais do que por comida ou sua imagem, mas pelo próprio espetáculo, pelo fantástico a ser compartilhado. Nesse circuito, que vai do real ao virtual e vice-versa - pois, como os autores bem pontuam, não há mais como separar uma versão on-line de outra off-line da vida -, o consumidor é convocado a gozar a fim de pôr em funcionamento toda uma máquina que “devora apaixonadamente os alimentos, não apenas com a boca, mas também com os olhos, polegares e ouvidos. Consome alimentos e imagens vinculadas a outras máquinas desejadoras, como câmeras, smartphones, fotos do Instagram, amigos, blogueiros, sites e empresas" (Kozinets et al., 2017, p. 672). Trata-se, ao final, do que é excessivo na própria convocação (Prado, 2013) ou agenciamento, no contexto de um tecnocapitalismo que vem transformando a natureza do consumo, das pesquisas sobre o consumidor e do marketing, assim como a busca humana por satisfação por meio do consumo.

0 estudo de Kozinets et al. (2017) ilustra bem a experiência de consumo como busca de satisfação pulsional que propus neste ensaio. Os autores não fazem uso da categoria "experiência de consumo", mas são numerosas as passagens no texto em que consideram a relação entre experiência, consumo e busca de satisfação excessiva. Algumas dessas passagens revelam que os autores estão cientes do uso mercadológico que esse conceito adquiriu, no sentido do quanto "uma mentalidade de design de experiência está subjacente ao entrelaçamento íntimo da tecnologia e do comportamento do consumidor" (p. 663). Outras referências apontam para um uso mais psicanalítico da concepção de experiência e sua relação com a pulsão, como quando argumentam que "a virtualidade da tecnologia gera possibilidades ilimitadas para novas experiências de corpo, e o desejo de chamar atenção atrai extremos de transgressões, a fim de criar um fluxo liberado de energia” (p. 675). Essa concepção de experiência apóia-se na teoria do desejo de Deleuze e Guattari $(1983,1987)$, que compreendem o desejo como energia e fluxo. No entanto, a psicanálise freudiana também ancora uma concepção de energia, por meio do conceito de pulsão. Em Freud, pulsão é fluxo constante de energia em busca de satisfação. É intensiva e sempre excessiva em sua circulação. O desejo forma-se, justamente, na barreira a essa busca de satisfação total. Assim, embora fosse necessária uma elaboração maior para estabelecermos as afinidades teóricas entre as concepções de desejo como energia, em Deleuze e Guattari, e de pulsão, em Freud, assim como de gozo, em Lacan, é possível afirmarmos que há uma profunda interlocução entre seus significados, conforme argumentado por Zizek (2011) e David-Ménard (2014). Portanto, quando Kozinets et al. (2017) afirmam que nossa atual rede tecnológica é a mais completa realização da ideia de desejo como um movimento energético incessante, podemos articular tal ideia com a do movimento da pulsão. Trata-se de uma máquina de produção de gozo, de uma "conexão coletiva apaixonada e direcionada comercialmente" que, segundo os autores, representa o estado atual da cultura de consumo (p. 679). Mas, se assumirmos a perspectiva psicanalítica enunciada neste ensaio, a promessa de gozo não significa sua entrega. Estruturalmente, mesmo em uma cultura que convoca seus sujeitos a gozar, o gozo pleno está interditado. Porém, o que acontece com a experiência do sujeito quando nada muda do ponto de vista da barreira ao gozo, mas, do ponto de vista de sua oferta, muda tudo? No plano subjetivo, se voltarmos à definição de pulsão como busca de satisfação plena, porém impossível, uma forma cultural estruturada em torno da promessa de gozo não possibilita mais nenhum apaziguamento, mesmo que temporário, do sujeito, pois quanto mais a satisfação pulsional é prometida, “mais vem a ser, tal como acontece em todos os mecanismos de adição, frustrada e, portanto, reiniciada” (Dufour, 2013, p. 386), caracterizando-se por uma compulsão à repetição. É justamente isso que alimenta a "rede de desejo", no sentido atribuído por Kozinets et al. (2017).

\section{Considerações finais sobre o curto-circuito da pulsão na experiência de consumo}

Ao longo deste ensaio, argumentei a favor de uma teorização da experiência de consumo como busca de satisfação pulsional ou gozo. Com base nisso, propus expandir o conceito de "experiência de consumo" para as análises das formas de consumo excessivas presentes na literatura dos estudos do consumidor, em especial, naquelas que têm se debruçado sobre o consumo virtual. Tais análises vêm mostrando o quanto essas formas de consumo têm investido em uma experiência de satisfação extrema.

Empurrar a pulsão para sua satisfação apresentava-se como transgressivo em formas culturais repressivas, daí a aposta de Deleuze e Guattari $(1983,1987)$ na emancipação humana por 
meio da liberação do desejo como fluxo. Porém, em uma cultura do gozo, essa pulsão entra em curto-circuito, a serviço de um capitalismo tecnológico de consumo que coloca a transgressão em outro patamar, prometendo desterritorializar o desejo do corpo, apelando a um sem-limite, ao oferecer aos consumidores as possibilidades de se perder em um fluxo de imagens e fantasias, "de exibir a vida de alguém na rede e testemunhar sua reação, de consumir as exibições de mundos privados de outras pessoas exibidos em tempo real, de ser a primeira pessoa em suas redes a postar algo novo, prestes a se tornar viral" (Kozinets et al., 2017, p. 679).

Esse empuxo ao gozo tem resultado em mais consumo, expandindo, cada vez mais, sua esfera, assim como tem levado a processos de infinitas repetições. Para o campo dos estudos do consumidor, esse fenômeno coloca desafios epistemológicos, teóricos e éticos inéditos. Foi visando contribuir com esses desafios que propus compreender a experiência de consumo como busca de satisfação pulsional ou de gozo. Argumentei como a concepção de experiência de consumo acabou surgindo em um contexto histórico que lhe permitiu servir a um modo de funcionamento de um marketing experiencial voltado a produzir formas de satisfação cada vez mais intensas que continuaram se acelerando com as novas tecnologias da informação. Com a teoria pulsional, fui além da compreensão fenomenológica da experiência de consumo, indicando o quanto essa satisfação pode não resultar em algo prazeroso ou divertido, em especial, quando se é impelido constantemente a essa busca de satisfação extrema. De uma perspectiva ética, questionei as consequências dessa busca de satisfação em uma forma cultural que apela ao excessivo e, assim, cheguei às análises contemporâneas sobre consumo on-line em redes/mídias sociais, que têm exaltado o engajamento apaixonado do consumidor, sem uma discussão mais crítica sobre suas consequências. Os pesquisadores do campo do consumo, alertam Kozinets et al. (2017), devem manter os olhos bem abertos e olhar corajosamente para esse abismo ontológico e axiológico que esses novos agenciamentos tecnológicos vêm produzindo. Para isso, o conceito de experiência de consumo torna-se fundamental, desde que retirado da caixinha dos prazeres considerados positivos.

\section{REFERÊNCIAS}

Abott, L. (1955). Quality and competition. New York, USA: Columbia University Press.

Alderson, W. (1957). Marketing behavior and executive action. Homewood, USA: Irwin.
Arnould, E., \& Price, L. (1993, June). River magic: Extraordinary experience and the extended service encounter. Journal of Consumer Research, 20, 24-45. doi: 10.1086/209331

Bardhi, F., \& Eckhardt, G. (2017, October 1). Liquid consumption. Journal of Consumer Research, 44(3), 582-597. doi: 10.1093/jcr/ucx050

Belk, R., \& Costa, J. (1998, December). The mountain myth: A contemporary consuming fantasy. Journal of Consumer Research, 25 , 218-240. doi: $10.1086 / 209536$

Campbel, C. (2007). Romanticism, introspection and consumption: A response to professor Holbrook. Consumption, Markets and Culture, 1(2), 165-173. doi: 10.1080/10253866.1997.9670296

Carù, A., \& Cova, B. (2003). Revisiting consumption experience: A more humble but complete view of the concept. Marketing Theory, 3(2), 267-286. doi: 10.1177/14705931030032004

Copjec, J. (1994). Read my desire: Lacan against the historicists. Cambridge, USA: The MIT Press.

David-Ménard, M. (2014). Deleuze e a psicanálise. Rio de Janeiro, RJ: Civilização Brasileira.

Deleuze, G., \& Guattari, F. (1983). Anti-Oedipus: Capitalism and schizophrenia. Minneapolis, USA: University of Minnesota Press.

Deleuze, G., \& Guattari, F. (1987). A Thousand Plateaus: Capitalism and Schizophrenia, Minneapolis: University of Minnesota Press.

Dufour, D.-R. (2013). A cidade perversa: Liberalismo e pornografia. Rio de Janeiro, RJ: Civilização Brasileira.

Firat, A., \& Dholakia, N. (1998). Consuming people: From political economy to theaters of consumption. New York, USA: Routledge.

Freud, S. (1996). Beyond the pleasure principle. In The standard edition of the complete psychological works of Sigmund Freud (Vol. 18). J. Strachey (Ed.). London, UK: Hogarth Press.

Freud, S. (2011). O mal-estar na civilização. São Paulo, SP: Penguin Classics/Companhia das Letras.

Freud, S. (2014a). As pulsões e seus destinos. São Paulo, SP: Autêntica.

Freud, S. (2014b). Compêndio de psicanálise. São Paulo, SP: Autêntica.

Gardner, B., \& Levy, S. (1955, March/April). The product and the brand. Harvard Business Review, 33, 33-39.

Gilovich, T., Kumar, A., \& Jampol, L. (2014). A wonderful life: Experiential consumption and the pursuit of happiness. Journal of Consumer Psychology, 25(1) (2015), 152-165. doi: 10.1016/j.jcps.2014.08.004

Hirschman, E., \& Holbrook, M. (1982, Summer). Hedonic consumption: Emerging concepts, methods and propositions. Journal of Marketing, 46(3), 92-101. doi: 10.1177/002224298204600314

Holbrook, M. (2006, December). The consumption experience: Something new, something old, something borrowed, something sold - Part 1, Review. Journal of Macromarketing, 26(2), 259-266. doi:10.1177/0276146706291064

Holbrook, M., \& Hirschman, E. (1982, September). The experiential aspects of consumption: Consumer fantasies, feelings and fun. Journal of Consumer Research, 9(2), 132-140. doi: 10.1086/208906

Ianninni, G. (2014). Epistemologia da pulsão: Fantasia, ciência, mito. In S. Freud, As pulsões e seus destinos. Belo Horizonte, MG: Autêntica.

Jameson, F. (1998). The cultural turn: Selected writings on the postmodern, 1983-1998. London, UK: Verso. 
Jantzen, C., Fitchett, J., Ostegaard, P., \& Vetner, M. (2012, June). Just for fun? The emotional regime of experiential consumption. Marketing Theory, 12(2), 137-154. doi: 10.1177/1470593112441565

Jay, M. (1994). Experience without a subject: Walter Benjamin and the novel. In M. Roth (Org.), Rediscovering history: Culture, politics and the psych (pp. 121-136). Stanford, USA: Stanford University Press.

Kozinets, R. (2002, June). Can consumers escape the market? Emancipatory illuminations from burning man. Journal of Consumer Research, 29, 20-38. doi: 10.1086/339919

Kozinetes, R., Patterson, A., \& Ashman, R. (2017). Networks of desire: How technology increases our passion to consume. Journal of Consumer Research, 43, 659-682. doi: 10.1093/jcr/ucwo61

Lacan, J. (1998). O seminário: Livro 11 - Os quatro conceitos fundamentais da psicanálise. Rio de Janeiro, RJ: Zahar.

Lanier, C., Jr., \& Rader, C. (2015, December). Consumption experience: An expanded view. Marketing Theory, 15(4), 407-508. doi: $10.1177 / 1470593115581721$

Lasch, S. (2006). Experience. Theory, culture and society, 23(2-3), 335 340. doi: 10.1177/026327640602300262

Lebergott, S. (1993). Pursuing hapiness: American consumers in the Twentieth Century. Princenton, USA: Princenton University Press.

Levy, S. (1959, July/August). Symbols for sale. Harvard Business Review, $37,117-119$.

Levy, S., \& Czepiel, J. (1974). Marketing and aesthetics: Proceedings, Chicago, USA: American Marketing Association.

Loose, R. (2015). The other side of marketing and advertising: Psychoanalysis, art and addiction. Marketing Theory, 15(I), 31-38. doi: $10.1177 / 1470593114558527$
McGowan, T. (2004). The end of dissatisfaction? Jacques Lacan and the emerging society of enjoyment. Albany, USA: State University of New York Press.

Pine, J., \& Gilmore, J. (1999). The experience economy: Work is theatre and every business a stage. Harvard, USA: HBS Press.

Prado, J. (2013). Convocações biopolíticas dos dispositivos comunicacionais. São Paulo, SP: Educ/Fapesp.

Raymen, T., \& Smith, O. (2017). Lifestyle gambling, indebtedness and anxiety: A deviant leisure perspective. Journal of Consumer Culture, o(o) I-19. doi: 10.1177/1469540517736559

Safatle, V. (2007). A teoria das pulsões como ontologia negativa: Dossiê filosofia e psicanálise. Discurso, 36, 148-189.

Schmitt, B. (1999). Experiential marketing: How to get customers to sense, feel, think, act, and relate to your company and brands. New York, USA: Free Press.

Scott, R., Cayla, J., \& Cova, B. (2017). Selling pain to the saturated self. Journal of Consumer Research, 44, 22-43. doi: 10.1093/jcr/ucw071

Smith, O., \& Raymen, T. (2017). Shopping with violence: Black Friday sales in the British context. Journal of Consumer Culture, 17(3), 677694. doi: $10.1177 / 1469540515611204$

Woodward, M., \& Holbrook, M. (2013). Dialogue on some concepts, definitions and issues pertaining to 'consumption experience'. Marketing Theory, 13(3), 323-344. doi: 10.1177/1470593113485108

Zizek, S. (2002). For they know not what thet do: Enjoyment as a political fator. London, UK: Verso.

Zizek, S. (2011). Órgãos sem corpos: Deleuze e consequências. Rio de Janeiro, RJ: Cia. de Freud.

\section{CONTRIBUIÇÃO DA AUTORA}

A autora declara que realizou todas as etapas de desenvolvimento do estudo. Desde a conceitualização e abordagem teórica-metodológica, a revisão teórica (levantamento de literatura), coleta de dados, bem como na análise de dados e, por fim, redação e revisão final do artigo. 